\title{
Development of a Training System for Cardiac Muscle Palpation
}

\author{
Tatsushi Tokuyasu ${ }^{1}$, Shin'ichiro Oota ${ }^{1}$, Ken'ichi Asami $^{1}$, Tadashi Kitamura ${ }^{1}$, \\ Gen'ichi Sakaguchi², Tadaaki Koyama ${ }^{2}$, and Masashi Komeda ${ }^{2}$ \\ ${ }^{1}$ Kyushu Inst. of Tech., Dept. of Mechanical Systems Eng., \\ 680-4 Kawazu, Iizuka, Fukuoka 820-8502, Japan \\ \{toku, oota, asami, kita\} @imcs.mse.kyutech.ac.jp \\ http: //www.imcs.mse.kyutech.ac.jp/ \\ ${ }^{2}$ Kyoto Univ., Dept. of Cardiovascular Surgery, Konoe-cho, Yoshida, \\ Sakyo-ku, Kyoto 606-8501, Japan \\ \{tadaakik, sakaguti, masakom\} @kuhp.kyoto-u.ac.jp \\ http: //www.kuhp.kyoto-u.ac.jp/ Cardiovasc-Surg/index.html
}

\begin{abstract}
Touching the cardiac muscle is necessary to get mechanical conditions of muscle before cardiac surgery. The cardiac palpation is the only way to make surgical plans for left ventricular plastic surgery. The training system for cardiac palpation we have developed consists of a MRI-based virtual left ventricular image and a one-dimensional manipulator as a haptic device. Mechanical properties of the cardiac muscles of a dog and a pig are embedded in the virtual heart. Our experiments show that the developed training system enables users to feel the reactional force to the virtual heart surface from the manipulator in real time.
\end{abstract}

\section{Introduction}

In order to put into a surgical operation for ventricular plastic surgery, the cardiac surgeon needs to touch the cardiac muscle to recognize where thin and soft regions of the muscular wall due to myocardial infraction and dilate cardiomyopathy are located. Qualitative estimation of partial geometric properties for the heart can be made from cardiac medical image data such as MRI, UT and XCT, which are available in the diagnostic process before the operation. But any medical imaging techniques and instruments provide neither mechanical information nor satisfactorily wall thickness information of the diseased ventricle. Namely, the opportunity to inspect the beating heart based on feels is limited to the scene of the operating room. Therefore training systems for cardiac muscle palpation are desired to the cardiac surgeon.

In this study, a virtual heart model is proposed to design and build a training system for the cardiac muscle palpation. We focus on the importance of dynamic mechanical properties of the cardiac muscle that is reflected on a $3 \mathrm{D}$ image of the left ventricle interactively responding to the user's finger force. Thus the proposed training system enables to (1) visualize the virtual heart model including the mechanical characteristics for cardiac muscle, and (2) feel the elasticity of the virtual heart through the haptic-device to transmit the reactional force to the finger. The goal of our system initially offer an opportunity for inexperienced surgeons to palpate the cardiac muscle is provided. The proposed training system would make a benefit of the clinical 
use if either real measurements or estimates of mechanical properties of each individual diseased heart are available. This paper reports the modeling of the virtual heart and determining the mechanical parameters of the heart and discusses these techniques.

In the following section, the system structure of the palpation training system is described. In the third section, the method of modeling the virtual left ventricle is presented. In the fourth section, the manipulator structure for transmitting the elasticity of the virtual heart is presented. In the fifth, sixth and final sections, experimental results, discussions and conclusions will be given respectively.

\section{System Descriptions}

Fig.1 shows the schematic diagram of the proposed training system for cardiac palpation, which consists of two PCs and the haptic device. The one computer processes the virtual heart motion based on the dynamic model graphically with use of OpenGL graphics library. The other computer controls and measures the values of electric current of the manipulator through AD/DA conversion board, where the input-output data are processed in real time. These two PCs are connected via a data communication board and a one-dimensional manipulator as a haptic-device. The sampling rate of the control drive is $1000[\mathrm{~Hz}]$.

The virtual heart model is connected to a simple systemic circulatory system model as Windkessel model. The left atrial pressure of $9[\mathrm{mmHg}]$, the right atrial pressure of 7 [mmHg], and the means of aortic pressure of 100 [mmHg] are assumed as the normal condition of the virtual heart. The vascular resistance depends on the body weight. Here the body weight is assumed to be $25[\mathrm{~kg}]$ of the dog. The trainee can adjust these parameters depending on the situations.

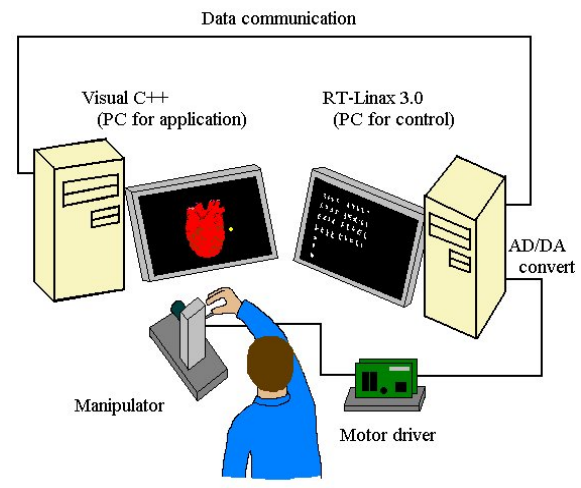

Fig. 1. Schematic diagram of the training system for cardiac palpation

\section{$3 \quad$ Virtual Heart Modeling}

\subsection{Graphical Modeling}

The virtual heart is built using the human MRI image in the textbook [1]. The MRI image includes the wire graphics for the left ventricle over 5 time frames from end- 
diastole to end-systole. We expanded the 5 time frames in the 21 time frames, and made the movie image of the virtual beating heart. The 21 time frames include the 8 frames of contractile period and the 13 frames of relaxational period, and both ratios are 1:1.7 because of the correspondence to the pulsational duration. In order to draw the smooth curve for the surface of heart, the method of a spline interpolation is applied to the virtual heart graphics.

\subsection{Mechanical Modeling}

The mechanical model for the virtual heart is developed for the real time linkage between the virtual and actual motions. Since the mechanical modeling of 2 th order allows computing the possible behavior of cardiac muscular surface in the case of giving an external force, a method of simulating some complex shape such as F.E.M. is avoided. Here the patch is defined as the part where the finger pushes the virtual heart. The dynamic model is assigned to the patch. Fig. 2 shows the schematic diagrams of dynamic and formal models for cardiac muscle, where the dynamic model is composed of mass, spring, and damper as the muscle element. The previous muscular models [2] which compose of parallel damper and spring are unsuitable to analyze the ventricular pressure data of a dog for identifying the mechanical parameters, so that more complex cardiac model is adopted in the horizontal muscle model. In addition, the contractile elements depending on a canine cardiac elastance are used in the horizontal direction.

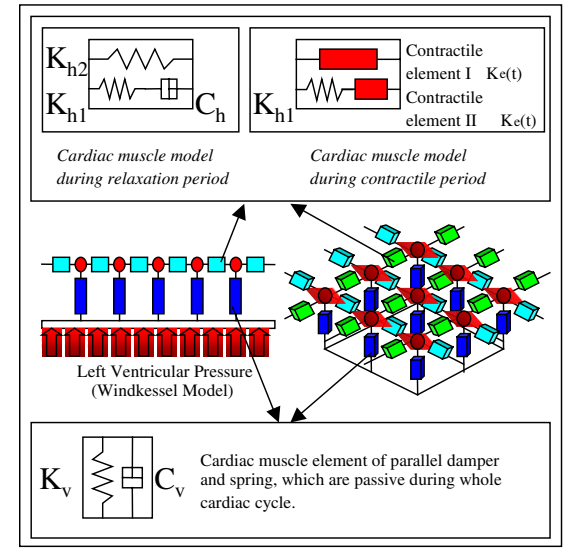

Fig. 2. Schematic diagrams of dynamic models for cardiac muscle

\subsection{Mechanical Parameters of Vertical Element}

The mechanical coefficients in the vertical direction to the heart surface are measured from the cardiac muscle extracted from a pig by using the lab-made instrumental for position and force instrument. The whole weight of a pig's heart is $310[\mathrm{~g}]$. A labmade instrument is composed of two arms of aluminum, an angular sensor, and a force sensor. The method how to measure the cardiac characteristics is to fasten the left ventricle to the force sensor attached on the arm. The measured values represent 
the elasticity $\mathrm{K}_{\mathrm{v}}[\mathrm{N} / \mathrm{m}]$ for the certain ventricular region. When the finger pushes the virtual heart, the reactional force is obtained at the contact area.

The viscosity $\mathrm{C}_{\mathrm{v}}[\mathrm{Ns} / \mathrm{m}]$ is identified by a simple rapid-released technique, where the restoration time from the depression is measured and simulated by using the vertical muscle model. The value of $\mathrm{C}_{\mathrm{v}}$ is obtained from restoration time of the depression by a stick.

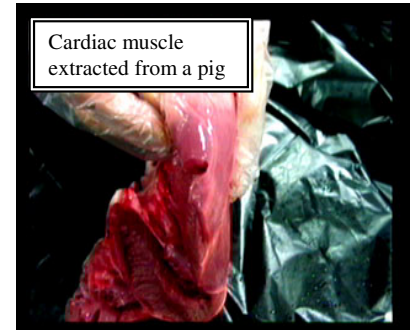

(a) Elasticity measurement

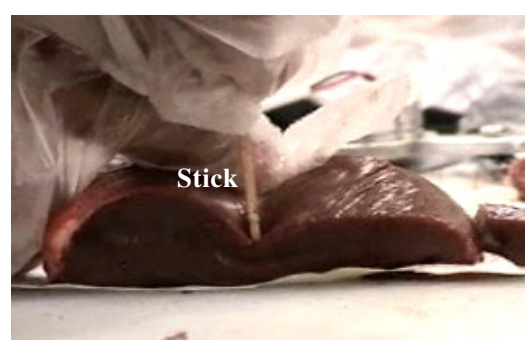

(b) Viscosity measurement

Fig. 3. Measurements of elasticity and viscosity for vertical elements of cardiac muscle of a pig

\subsection{Mechanical Parameters of Horizontal Element}

The mechanical characteristics in the horizontal direction to the heart surface are measured from the beating heart of an anesthetized dog in the experimental animal room at Kyoto University. The body weight of the dog is about $25[\mathrm{~kg}]$. The mechanical coefficients in the horizontal direction are important for the virtual heart because they govern the whole feature of the virtual heart. As the cardiac characteristics, the left ventricular pressure, left atrial pressure, and the cardiac output are measured from the left ventricle restrained by the same lab-made force and position measurement instrument. The beating motion is captured by the digital video tape recorder (Fig.4 (a)). By using the cardiac muscular contraction and the cardiac output data, the volume of one pulsation for the left ventricle is estimated. Fig.4 (b) shows the pressurevolume diagram plotted by using the measured data.
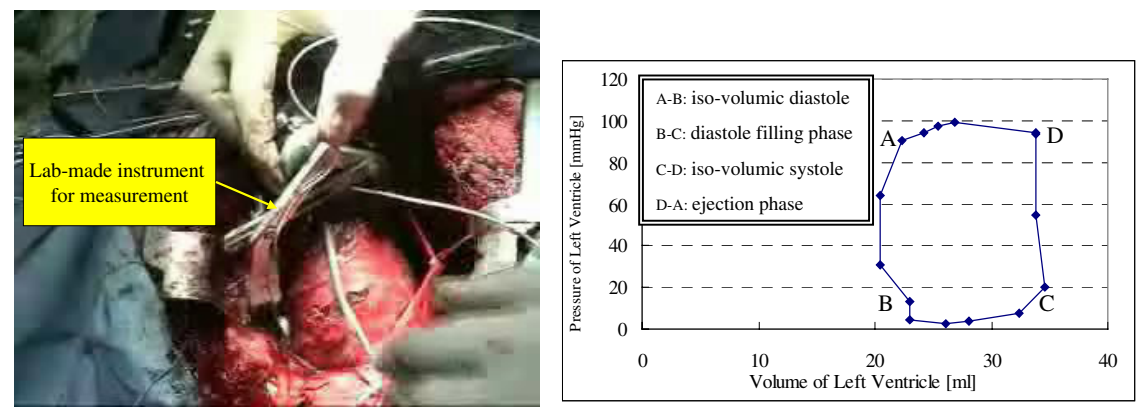

(a) Measurement state

(b) Pressure-Volume diagram from an actual data

Fig. 4. Results of measurement for the cardiac characteristics from an anesthetized dog

The cylindrical model for the horizontal element is developed for the left ventricle because the measured data depend on the whole characteristics of the left ventricle. 
Fig.5 (a) shows the schematic diagrams of the cylinder model of the left ventricle during relaxation phase, and (b) for contraction one.

By using the data of the left ventricular pressure during relaxation phase, so that the mechanical coefficients in the horizontal element are determined in the model (a). In the models of (a) and (b), a change of the left ventricular volume is given by the following equation (1).

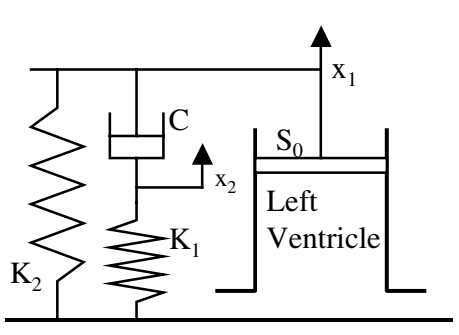

(a) During relaxation phase

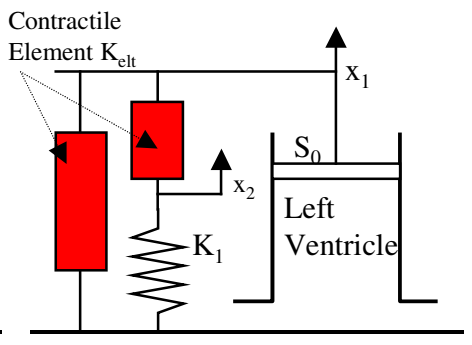

(b) During contraction phase

Fig. 5. Schematic diagrams of the cylindrical model of the left ventricle

$$
\dot{V}_{L V}(t)=S_{0} \dot{x}_{1}
$$

The $\mathrm{x}_{2}$ is dynamic during iso-volumic relaxational phase. For the series element composed of damper $\mathrm{C}$ and spring $\mathrm{K}_{1}$ in the model of (a), the balance of force derives the equation (2).

$$
P_{L V}(t)=P(0) e^{-\frac{K_{1}}{C} t} .
$$

Since both $\mathrm{x}_{1}$ and $\mathrm{x}_{2}$ are dynamic during diastolic filling phase, the calculated elasticity is given by the following equation (3).

$$
\begin{aligned}
& f(t)=-K_{2} x_{1}-C\left(\dot{x}_{1}-\dot{x}_{2}\right) . \\
& J\left(t, K_{1}, K_{2}\right)=\int_{0}^{t}\left(f(t)-\hat{f}\left(t, K_{1}, K_{2}\right)\right)^{2} d t .
\end{aligned}
$$

In order to determine horizontal parameters, the mean square error method is applied under the criterion to be minimized equation (4). The above techniques for the identification are applied to the measured data of the base, middle, and apex parts respectively. The calculated parameters are shown in the table 1 . Here the letter $\mathrm{v}$ indicates vertical and $\mathrm{h}$ for horizontal.

Table 1. Mechanical properties of dynamic model

\begin{tabular}{|l|c|c|c|}
\hline & Base & Middle & Apex \\
\hline $\mathrm{K}_{\mathrm{h} 1}:[\mathrm{N} / \mathrm{m}]$ & 30.1 & 164.8 & 98.9 \\
\hline $\mathrm{K}_{\mathrm{h} 2}:[\mathrm{N} / \mathrm{m}]$ & 766.5 & 709.6 & 655.4 \\
\hline $\mathrm{C}_{\mathrm{h}}:[\mathrm{Ns} / \mathrm{m}]$ & 4.6 & 2.1 & 8.2 \\
\hline $\mathrm{K}_{\mathrm{v}}:[\mathrm{N} / \mathrm{m}]$ & 90 & 75 & 45 \\
\hline $\mathrm{C}_{\mathrm{v}}:[\mathrm{Ns} / \mathrm{m}]$ & 72 & 60 & 51 \\
\hline
\end{tabular}


During contractile phase, spring and damper are replaced to the contractile element, which depends on the elastance for the left ventricle. The effective area determined from the equation (5) shows the spatial reduction of the patch in the left ventricle during contractile phase. Finally the spring of the contractile element is determined by the equation (6). Here parameter $\alpha$ is a compensational coefficient and is given in accord with the part of the diseased heart.

$$
\begin{aligned}
& S_{n}(t)=\frac{1}{T_{S}} \int\left(\frac{V_{e}(t)}{L_{0}(t)}\right) d t . \\
& K_{e}(t)=\alpha S_{n} S(t) E_{l t}(t) .
\end{aligned}
$$

The meanings of the variables are shown as follows,

$$
\begin{aligned}
& \mathrm{T}_{\mathrm{s}}: \text { Contractile period }[\mathrm{s}], \\
& \mathrm{V}_{\mathrm{e}} \text { : Effective volume of the left ventricle }[\mathrm{ml}], \\
& \mathrm{L}_{0}: \text { : Effective length of the left ventricle }[\mathrm{m}], \\
& \mathrm{S} \text { : The contact area of virtual finger }\left[\mathrm{m}^{2}\right], \\
& \mathrm{E}_{\mathrm{lt}} \text { : Elastance of the left ventricular muscle }[\mathrm{mmHg} / \mathrm{ml}] .
\end{aligned}
$$

\section{Manipulator for Force Feedback}

In this training system, the force between the virtual heart and the user's finger is transmitted through the manipulator. The trainee can touch the virtual heart with operating the manipulator and get the pulsatile elasticity of the cardiac muscle. The previous work shows the technique for transmitting the minute torque [3]. The method of hybrid control achieves to transmit the minimum force of 5 [gf]. Fig.6 shows the overview of the manipulator, which is composed of a DC servomotor, a single lever, and an angular sensor. This training system converts the circular movement of the lever into the straight movement in the computational graphics.

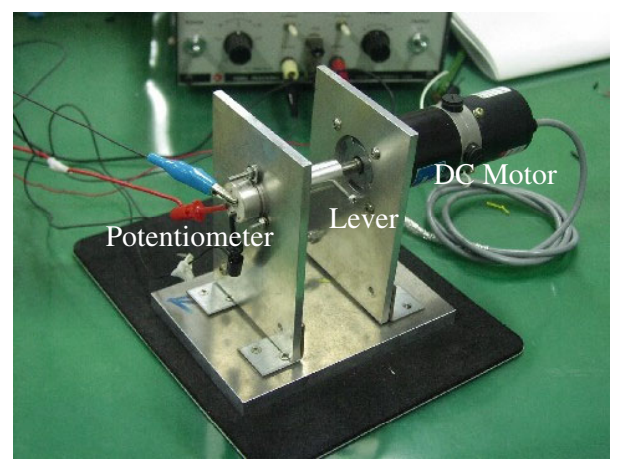

Fig. 6. Manipulator for force transmission

\section{Results}

Fig.7 (a) shows the graphics image of the virtual left ventricle on the computer display. The trainee is able to rotate and expand the virtual heart by changing the view- 
point. For the patch, he/she can push the affected part by the virtual finger through the manipulator. The patch becomes depressed when the virtual heart is pushed $8[\mathrm{~mm}]$. Fig.7 (b) shows the diagram for pressure and volume of the virtual heart, where the one graph shows the natural condition of the virtual heart for one pulsation and the other graph for when the virtual finger pushes the virtual heart. For the relaxational phase, the left ventricular pressure does not increase because the left atrium pressure is fixed. As the result, the cardiac output decrease in about 74 [\%] when the virtual heart is pushed $8[\mathrm{~mm}]$.

Fig. 8 shows the performance for the response of the palpation system. In accord with the displacement of the manipulator operation, the reactional force from the virtual heart is obtained with calculating the beating elasticity of the cardiac muscle. In this experiment, it is assumed that the cardiac palpation is carried out under the low ventricular pressure by adjusting the input value for the left atrial pressure, because the high ventricular pressure prevents feeling the elasticity of the cardiac muscle. The first author Tokuyasu recognizes that the feel of pressing the virtual heart is close to his feel for the dog heart we used for the experiment.

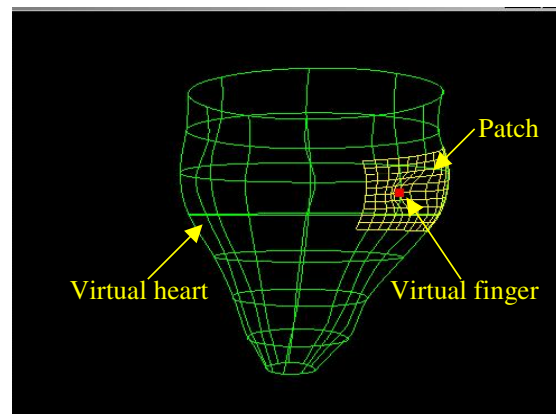

(a) Graphic image

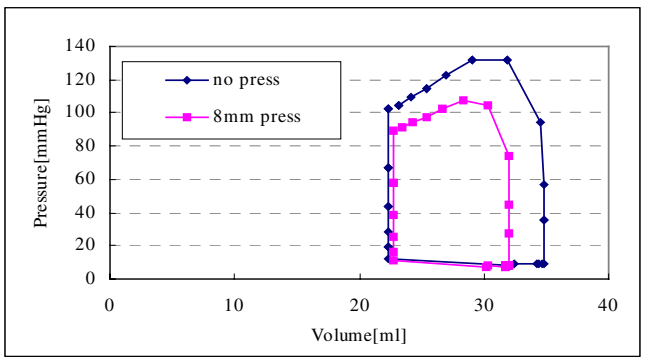

(b) Pressure-Volume diagram

Fig. 7. Experimental responses of the virtual heart

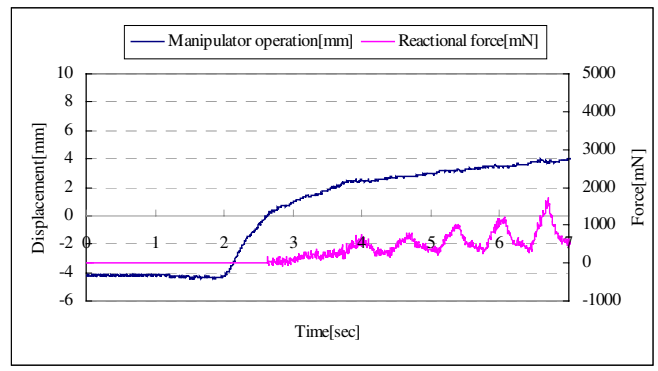

Fig. 8. Mechanical response of the palpation system

\section{Discussions}

The cardiac palpation is important for the surgeon to make a surgical plan on the basis of feels of the diseased heart. Any diagnostic methods and instruments do not provide mechanical information about the heart so enough to make a surgical plan as palpa- 
tion on the operative scene. Therefore the mechanical modeling and the training system for cardiac palpation are strongly desired from cardiac surgeons. Thus the proposed training system with the virtual left ventricle has an advantage of cardiac plastic surgery.

Modeling the whole heart consisting of left and right ventricles and atriums are desired to get better virtual reality for the more practical palpation training system. But there was a problem of determining the mechanical parameters because of the limitation of measurements of real hearts by the measuring instrument. To solve this problem, the mechanical parameters should be measured for more and smaller spots on the cardiac muscle with a smaller force-measuring probe. The proposed modeling technique of the left ventricle facilitates to create infarcted regions with high compliance by changing the spring constants.

For clinical applications, a knowledge database for estimating mechanical parameters of each individual diseased heart will be needed reflecting the surgeon's experience about the correspondence between the mechanical characteristics of past patients' hearts and their feels. MR-elastography [4], which can non-invasive measure elastic properties of a living tissue, if completed, would immediately enable our system to be used for planning the surgery of each individual patient before the operation.

The one-dimensional manipulator allows the finger only to push the patch. However the actions to rub and hold the heart are necessary to simulate real cardiac palpation. Therefore the development of a manipulator will be needed to detect the motion of plural fingers and feedback their forces to the fingers.

\section{Conclusions}

We undertook the development of the palpation system in accord with the cardiac surgeon's request. The virtual heart model provides the real time response modeling a local area of the left ventricle in terms of the dynamic model for the cardiac muscle. The obtained force from the manipulator is corresponding to the feels of the canine cardiac elastance we used for the measurement. These results satisfy basic required conditions for the development of the palpation system. The functions to be added to the palpation system include modeling of the blood flow due to the large cardiac deformation by touching. Moreover the multiple touching points on the virtual heart and the haptic device corresponding plural fingers should be added.

\section{References}

1. Dimitris N. Metaxa, Physical-based Deformable Models Applications to Computer Vision, Graphics and Medical Imaging, page 211, 1997.

2. T.Tokuyasu,. et al., Development of A Training System for Cardiac Muscle Palpation with Real-Time Image Processing and Force Feedback, Proc. JCAS The 10th Japan Society of Computer Aided Surgery, pp.129-130, 2001.

3. T.Tokuyasu, T.Kitamura, A study on Minute Torque Transmission for Tele Microsurgery. Proc. of JSME Robomec2001, CD-ROM, 2001.

4. Muthupillai R, and Ehman RL., Magnetic resonance elastography, Nat Med 2:601-603b, 1996. 